\title{
Die diagnostische Bedeutung der Lipid-Elektrophorese für die Beurteilung von Störungen des Lipid-Stoffwechsels
}

\author{
Von \\ G. BERG \\ Aus der Medizinischen Klinik mit Poliklinik der Universität Erlangen-Nürnberg \\ (Direktor: Prof. Dr. N. Henning)
}

(Der Schriftleitung zugegangen am 22. September 1962)

\begin{abstract}
Durch Lipidelektrophorese lassen sich die Lipidanteile der Serumfraktionen erfassen. Gegenüber der Papierelektrophorese bietet die Dünnschichtelektrophorese auf Stärke insofern Vorteile, als sie die Trennung größerer Serummengen und damit die Darstellung der Fraktionen mit geringem Lipidgehalt ermöglicht. Mit dieser Methode lassen sich Lipide in der Albumin-Bande nachweisen, die zur Gruppe der $\alpha_{1}$-Lipoproteide gehören. Der Gehalt an Lipiden der eigentlichen $\alpha_{1}$-Fraktion ist gering. Die $\alpha_{2}$-Fraktion enthält regelmäßig Lipide, ihr Hauptanteil ist in $\operatorname{der} \beta$-Fraktion nachzuweisen. Die $\gamma$-Globulin-Fraktion ist frei von Lipiden.

Bei der akuten Hepatitis ist die $\alpha_{1}$ - und $\alpha_{2}$-Lipid-Fraktion erniedrigt, die $\beta$-Fraktion erhöht. In Fällen von chronischer Hepatitis sind die $\alpha$-Fraktionen oft erhöht, desgleichen bei nachgewiesener Leberepithelverfettung. Hohe $\alpha_{2}$ - und $\beta$-Lipidwerte finden sich bei essentieller Hyperlipämie, Atherosklerose und Nephrose.

Das Neutralfett wird in Form von Chylomikronen hauptsächlich in der $\alpha_{2}$-Fraktion transportiert, wie vergleichende Untersuchungen vor und nach Eliminierung leichter Lipoproteide durch Ultrazentrifugierung ergeben haben. In manchen Fällen findet sich aber auch nach Entfernung leichter Lipoproteide ein hoher Lipidanteil im Bereich der $\alpha_{2}$-Fraktion. In diesen Fällen kommt es nach Fettzufuhr zu einem weiteren Anstieg dieser Fraktion, die Fettklärung ist verzögert. Dieses Phänomen ist Ausdruck einer Fett-Transportstörung und ist besonders bei Fällen von essentieller Hyperlipämie, chronischer Hepatitis, Leberepithelverfettung und Nephrose zu beobachten.
\end{abstract}

The lipid componerits of serum fractions can be demonstrated byy.lipid electrophoresis. Compared with paper electrophoresis, thin layer electrophoresis on starch has the advantage that larger amounts of serum can be analysed and the fractions of low lipid content can be shown. The $\alpha_{1}$-lipoproteins in the albumin band can be demonstrated by this method. The lipid content of the $\alpha_{1}$-fraction is low. The $\alpha_{2}$-fraction normally contains lipids and the $\beta$ fraction contains the largest proportion of lipids.

In acute hepatitis, the $\alpha_{1}$ and $\alpha_{2}$-lipid fraction is decreased and the $\beta$-fraction increased. In chronic hepatitis and in fatty liver epithelium, the fractions are often increased. High $\alpha_{2}$ and $\beta$-lipid values are found in essential hyperlipaemia, atherosclerosis and nephrosis.

Neutral fat is transported in the $\alpha_{2}$-fraction chiefly as chylomicrons, as shown by comparative studies before and after the elimination of light lipoproteins by ultracentrifugation. Often, however, a high lipid content is found in the region of the $\alpha_{2}$-fraction after the removal of light lipoproteins. In these cases, administration of fat causes a further increase in this fraction and lipid clearing is retarded. This phenomenon indicates a disturbance of fat transport and is especially marked in cases of essential hyperlipaemia, chronic hepatitis, fatty liver epithelium and nephrosis.

Die Lipid-Elektrophorese ist eine Sonderform der elektrophoretischen Serumanalyse, die die Bestimmung der Lipid-Anteile der Serumfraktionen ermöglicht. Zusammenfassende Darstellungen $(1,2,3,4)$, ausführliche Literatur bei Pezold (5). $\mathrm{Da}$ die Lipide an Proteine gebunden sind, wandern sie im elektrischen Feld und können durch unterschiedliche Wanderungsgeschwindigkeiten charakterisiert werden.

Nach papierelektrophoretischer Trennung lassen sich mit Fettfarbstoffen drei, gelegentlich auch vier lipidhaltige Eiweiß-Fraktionen darstellen. Teilt man den Streifen der Länge nach und färbt. die eine Hälfte mit Eiweiß- und die andere mit Fettfarbstoffen an, so findet sich die am schnellsten wandernde lipidhaltige Bande in der Albu$\min / \alpha_{1}$-Globulinfraktion. Der Hauptanteil der Serumlipide ist der $\beta$-Globulin-Fraktion zuzuordnen, eine weitere lipidhaltige Zone findet sich zwischen $\operatorname{der} \beta$ Fraktion und dem Start. Gelegentlich sind auch im Bereich der $\alpha_{2}$-Fraktion Lipide nachzuweisen.
Die Festigkeit der Lipid-Eiweiß-Bindung ist unterschiedlich. Bekanntlich werden bei der papierelektrophoretischen Trennung die Chylomikronen durch Adsorbtion von ihrer Trägerfraktion gelöst und wandern dann nicht mehr im elektrischen Feld. Sie bleiben zwischen der $\beta$-Fraktion und dem Start liegen (3). Aus diesem Verhalten ist auf eine sehr lockere Bindung der Chylomikronen an Proteine zu schließen. Diese Tatsache läßt die Papierelektrophorese zur Bestimmung der Transportfraktion für das Neutralfett und zum Nachweis von Störungen des Fett-Transportmechanismus ungeeignet erscheinen. Für die quantitative Auswertung der Lipide ist eine dem Gehalt streng proportionale Anfärbung aller Fettkomponenten unerläßlich. Quantitative Untersuchungen haben jedoch ergeben, daß sie sehr verschiedene Farbstoffinengen binden $(6,7)$. Das Neutralfett färbt sich am stärksten an, weniger gut das Cholesterin, am geringsten die Cholesterinester und die Phosphatide. Die Lipid-Elektrophorese ermöglicht daher keine quantitative Analyse der in den Serumfraktionen enthaltenen Lipide. Es wird lediglich die Relation der einzelnen Fraktionen bestimmt, wobei $\cdot \operatorname{der}$ Anteil der einzelnen Fettkomponenten unberücksichtigt bleibt. Für besondere Fragestellungen können einzelne Lipide (z. B. Phosphatide (8)) durch spezielle Nachweisverfahren dargestellt werden. Für die quantitative Bestimmung der Lipide in den Serumfraktionen ist nach wie 
vor die präparative Trennung als Methode der Wahl anzusehen. Schwierigkeiten für die quantitative Analyse ergeben sich aber auch aus dem geringen Serumlipidgehalt und der unterschiedlichen Menge der einzelnen Serumfraktionen. Die für eine papierclektrophoretische Analyse üblicherweise verwendeten Serummengen von $5 \mu l$ reichen nicht aus, Fraktionen mit geringem Lipidgehalt zu erfassen. Auch größere Mengen (bis $30 \mu l$ ) liefern noch keine befriedigenden Ergebnisse. Die Verwendung noch größerer Mengen scheitert aber an der begrenzten Trennkapazität des $\mathrm{Pa}$ piers. Die Fraktionierung wird bei diesen Mengen unzureichend (9).

Die Lipid-Elektrophorese auf Filterpapier ist daher nur mit Einschränkungen verwertbar. Sie gestattet die Abgrenzung einer schnellwandernden Lipidfraktion, die herkömmlicherweise als $\alpha$-Lipid-Fraktion bezeichnet wird und einer langsamer wandernden Komponente, $\operatorname{der} \beta$ Lipid-Fraktion. Die Neutralfette $z$ wischen $\operatorname{der} \beta$-Fraktion und dem Start, bleiben im allgemeinen unberücksichtigt. Bei der Auswertung werden damit lediglich Änderungen im Verhalten $\operatorname{der} \alpha$ - und $\operatorname{der} \beta$-Lipide erfaßt. Trotz dieser Einschränkungen sind zahlreiche Untersuchungen vorgenommen worden, die die Bestimmung der Lipidanteile der Serumfraktionen und ihr Verhalten unter normalen und pathologischen Bedingungen zum Gegenstand haben (5). Im Gegensatz zu den Veränderungen der Serumfraktionen bei den verschiedenartigen Erkrankungen lassen die Lipide oft keine entsprechende Befunde erkennen. Das gilt für die Gruppe der entzündlichen Erkrankungen und der malignen Prozesse. Während das Serumpherogramm hierbei erheblich verändert ist, wird das Lipidpherogramm zumeist normal gefunden (5). Die Verschiebungen der $\alpha$ und $\beta$-Fraktionen sind gering (10). Dagegen ist in anderen Fällen eine erhebliche Veränderung des Lipidgehaltes erkennbar, die nicht ohne weiteres aus dem Serumpherogramm zu vermuten ist. Schon frühzeitig wurde die oft mangelhafte Anfärbung- der $\alpha$-LipidFraktion bei Lebererkrankungen erkannt (zusammenfassende Literatur bei (5)). Als Ursache dieses Lipidschwundes wurde eine Verminderung oder ein Fehlen $\operatorname{det} \alpha$-Lipoproteidfraktion ermittelt (11). Die $\alpha$-Lipoproteide sedimentieren in der Ultrazentrifuge, es handelt sich also um schwere Lipoproteide (15). Es liegt nahe, in diesen Fällen eine Störung des Lipidstoffwechsels anzunehmen, dessen Ưrsache und Mechanismus jedoch im einzelnen noch nicht näher bekannt sind. $\mathrm{Da}$ derartige Befunde nur bei Lebererkrankungen vorkommen, sind sie diagnostisch verwertbar. Sie gehen im allgemeinen dem Schweregrad der Ërkrankung parallel. Bedeutsam ist auch die Zunahme der $\beta=$ Lipid-Fraktion, wie sie bei Leberparenchymerkrankungen, bei essentieller Hyperlipämie, in Fällen von Arteriosklerose ụnd besonders auch bei Nephrose beobachtet werden. Dieser Befund entspricht einer Zunahme "leichter Lipoproteide", die reich an Cholesterin und Neutralfetten sind (5). Sie sinḍ Ausdruck eines gestörten Fettstoffwechsels, der bei den genannten Krankheitsgruppen das gleiche Bild im Lipidpherogramm hervorruft.

Mit der Lipid-Elektrophorese kann man also verschiedene Arten eines gestörten Fettstoffwechsels erfassen und sie trotz der genannten Einschränkungen dia- gnostisch auswerten. Diese Auswertung erlaubt eine Einteilung in bestimmte Typen von Fettstoffwechselstörungen. Für den Kliniker ergibt sich die Möglichkeit, Änderungen dieser Stoffwechselsituation prognostisch auszuwerten, beispielsweise dann, wenn sich bei einer Lebererkrankung die anfänglich fehlende $\alpha$-Lipid-Fraktion normalisiert, oder wenn im Falle eines nephrotischen Syndroms eine extrem hohe $\beta$ Fraktion auf Normalwerte absinkt.

Einen wesentlichen methodischen Fortschritt erbrachte die Verwendung von Stärke als Trägermedium (12, 13, 14, 15). Sie gestattet die Trennung größerer Serummengen $(>1 \mathrm{ml})$, so $\mathrm{da}$ auch die geringeren Lipidanteile einzelner Serumfraktionen darzustellen sind.

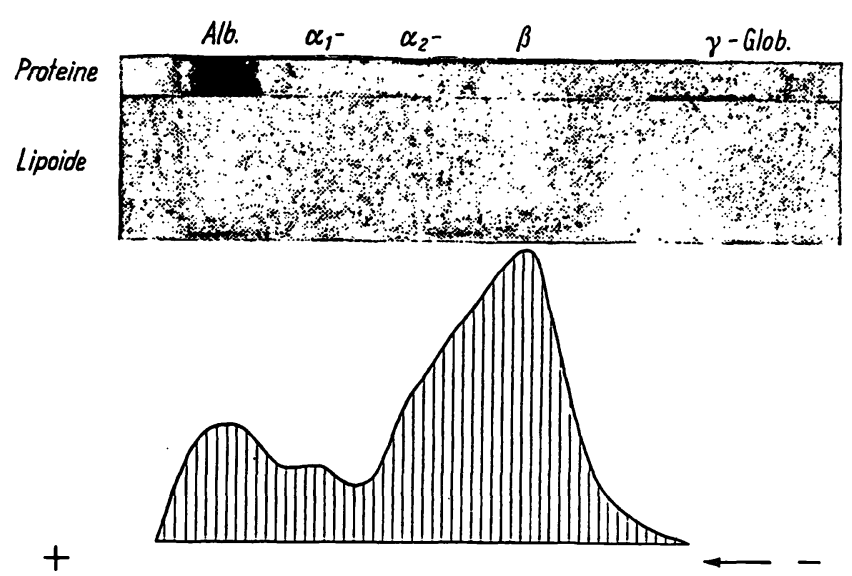

Abb. 1

Lipidpherogramm, Dünnschicht-Elektrophorese auf Stärke. Im Gegensatz zu der Papiermethode ist hier auch der Lipidanteil in $\operatorname{der} \alpha_{1}$, , besonders in der $\alpha_{2}$-Fraktion zu erkennen. Die $\gamma$-GlobulinFraktion ist lipidfrei.

Außerdem adsorbiert die Stärke keine Neutralfette, so $\mathrm{da}$ die lockere Bindung zu der Transportfraktion erhalten bleibt und dargestellt werden kann. Sowohl für praktisch-klinische wie für wissenschaftliche Untersuchungen ist deshalb die Dünnschichtelektrophorese auf Stärke als Methode der Wahl anzusehen (9). Die Serumtrennung wird auf einer $1-2 \mathrm{~mm}$ dicken Stärkeplatte vorgenommen, auf die ein trockener Filterpapierstreifen aufgepreßt wird, so daß das fraktionierte Serum in den Papierstreifen hineindiffundiert und angefärbt werden kann. Mit dieser Methode ist die schnellwandernde $\alpha$-Lipid-Fraktion eindeutig in die Albuminbande zu lokalisieren $(16,11)$. Der Lipidgehalt der $\alpha_{1}-$ Fraktion ist gering, in der $\alpha_{2}$-Globulin-Fraktion finden sich regelmäßig größere Lipidmengen. Auch mit der Dünnschichtelektrophorese findet sich die größte Lipidmenge in der $\beta$-Fraktion. Die $\gamma$-Globulin-Fraktion kann als frei von Lipiden gelten (9). Im Gegensatz zu der Papiermethode ist mit der Dünnschichtelektrophorese regelmäßig eine $\alpha_{2}$-Lipoproteidfraktion nachweisen (9, $13,17,18)$, dagegen fehlt die fetthaltige Zone zwischen der $\beta$-Fraktion und dem Start. Der Beweis dafür, daß der Neutralfetttransport in der $\alpha_{2}$-Fraktion erfolgt, ist durch ein einfaches Experiment zu erbringen. Werden die leichten Lipoproteide (Chylomikronen) 'durch 
Ultrazentrifugierung von der Transportfraktion getrennt, so daß sie sich als Fettschicht an der Oberfläche des Zentrifugierröhrchens ansammeln, so ist im Lipidpherogramm die $\alpha_{2}$-Fraktion deutlich erniedrigt (Abb. 2), (19). Manche Seren weisen auch _nach Ultra-

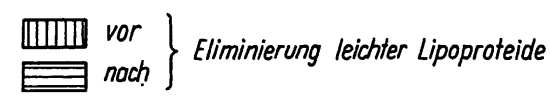

a Typ bei normoler Klọrung

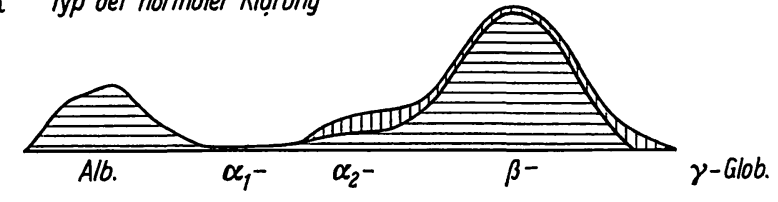

b Typ bei Klörinsuffizienz

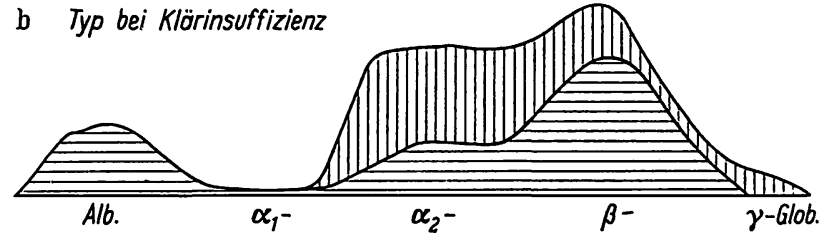

Abb. 2

zentrifugierung einen hohen Lipidgehalt in $\operatorname{der} \alpha_{2}-$ Fraktion auf (19). Auch mit der Papiermethode findet man hierbei häufig eine $\alpha_{2}$-Lipoproteid-Fraktion. Nach Fettbelastung steigt in diesen Fällen der Lipidgehalt der $\alpha_{2}$-Fraktion weiterhin an, die Fettklärung ist verzögert. Dieses Phänomen ist Ausdruck einer Fetttransportstörung (19). Es ist in Fällen von Arteriosklerose, Nephrose und Leberepithelverfettung zu beobachten, also in den Fällen, wo bekanntermaßen eine Störung des Fettstoffwechsels vorliegt. In diesen Fällen ist eine Mobilisierung von Transportfraktionen für leichte Lipoproteide anzunehmen, weil die Klärung verzögert abläuft. Es ist aber auch zu erwägen, ob hier nicht eine besonders feste Bindung der Chylomikronen an Lipo-

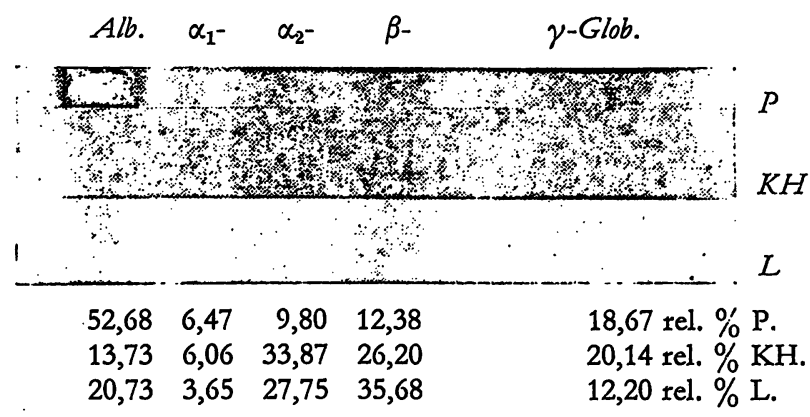

Abb. 3. Dünnschichtelektrophorese auf Stärke

\section{N. +69 Jahre. Arteriosklerose}

Im Proteinpherogramm ist eine Verminderung der AlbuminFraktion und eine geringe Zunahme der $\alpha_{2}-$ und $\gamma$-Globuline festzustellen. Die Kohlenhydratanteile $\operatorname{der} \alpha_{1}$-Globulin-Fraktion sind vermindert, die der $\alpha_{2^{-}}, \beta$ - und $\gamma$-Globulin-Fraktionen erhöht. Im Lipidpherogramm ist eine Zunahme der $\alpha_{2}$-GlobulinFraktion auffallend.

Bemerkenswert ist die $\alpha_{2}$-Globulinzunahme, die durch Glykoproteide und besonders durch Lipoproteide bedingt ist. proteide vorliegt (20). Bemerkenswert ist, daß hierbei nicht nur Veränderungen der Lipidanteile der Serumfraktionen festzustellen sind. Es lassen sich darüber hinaus auch pathologische Verschiebungen der Proteinwie Kohlenhydratanteile nachweisen.

Die Lipid-Elektrophorese ermöglicht im übrigen die Beurteilung pathologischer Serumveränderungen. Zwischen den Proteid- und den Lipidanteilen der Serumfraktionen bestehen bestimmte gesetzmäßige Beziehungen (21), die sich durch Elektrophorese nachweisen lassen und die den Ergebnissen chemischer Analysen entsprechen.

Tab. 1

Prozentualer Anteil der Serum-Lipide und -Proteine bei verschiedenen Erkrankungen. ( $a=$ Proteine; $b=$ Lipide; statistisch gesicherte Unterschiede kursiv).

\begin{tabular}{|c|c|c|c|c|c|c|c|}
\hline \multirow{2}{*}{ Diagnose } & \multirow{2}{*}{\multicolumn{2}{|c|}{$\begin{array}{c}\text { Zahl } \\
\text { d. } \\
\text { Fälle }\end{array}$}} & \multirow{2}{*}{$\begin{array}{l}\text { Albu- } \\
\text { min }\end{array}$} & \multicolumn{4}{|c|}{ Globuline } \\
\hline & & & & $\alpha^{1}$ & $\alpha^{2}$ & $\beta$ & $\gamma$ \\
\hline Normalbefunde & $\begin{array}{l}\text { a) } \\
\text { b) }\end{array}$ & $\begin{array}{l}99 \\
38\end{array}$ & $\begin{array}{l}58,9 \\
20,2\end{array}$ & $\begin{array}{l}6,2 \\
3,9\end{array}$ & $\begin{array}{r}8,4 \\
14,6\end{array}$ & $\begin{array}{l}11,5 \\
51,9\end{array}$ & $\begin{array}{r}13,9 \\
8,9\end{array}$ \\
\hline EntzündlicheProzesse & $\begin{array}{l}\text { a) } \\
\text { b) }\end{array}$ & $\begin{array}{l}38 \\
14\end{array}$ & $\begin{array}{l}46,8 \\
16,1\end{array}$ & $\begin{array}{l}8,0 \\
4,5\end{array}$ & $\begin{array}{l}11,8 \\
15,4\end{array}$ & $\begin{array}{l}13,5 \\
58,2\end{array}$ & $\begin{array}{r}18,5 \\
6,3\end{array}$ \\
\hline MaligneErkrankunger & b) & $\begin{array}{l}47 \\
24\end{array}$ & $\begin{array}{l}48,6 \\
19,3\end{array}$ & $\begin{array}{l}8,3 \\
4,1\end{array}$ & $\begin{array}{l}11,8 \\
13,4\end{array}$ & $\begin{array}{l}12,9 \\
58,7\end{array}$ & $\begin{array}{r}18,3 \\
6,4\end{array}$ \\
\hline Akute Hepatitis & $\begin{array}{l}\text { a) } \\
\text { b) }\end{array}$ & $\begin{array}{l}27 \\
10\end{array}$ & $\begin{array}{l}52,9 \\
10,3\end{array}$ & $\begin{array}{l}6,5 \\
3,1\end{array}$ & $\begin{array}{l}8,1 \\
8,9\end{array}$ & $\begin{array}{l}12,1 \\
68,5\end{array}$ & $\begin{array}{r}20,3 \\
9,0\end{array}$ \\
\hline Chronische Hepatitis & $\begin{array}{l}\text { a) } \\
\text { b) }\end{array}$ & $\begin{array}{r}17 \\
9\end{array}$ & $\begin{array}{l}49,3 \\
21,5\end{array}$ & $\begin{array}{l}6,5 \\
3,2\end{array}$ & $\begin{array}{r}8,4 \\
15,2\end{array}$ & $\begin{array}{l}12,2 \\
51,2\end{array}$ & $\begin{array}{r}23,5 \\
4,5\end{array}$ \\
\hline Arteriosklerose & $\begin{array}{l}\text { a) } \\
\text { b) }\end{array}$ & $\begin{array}{l}17 \\
18\end{array}$ & $\begin{array}{l}50,3 \\
17,2\end{array}$ & $\begin{array}{l}7,9 \\
4,5\end{array}$ & $\begin{array}{l}13,1 \\
15,3\end{array}$ & $\begin{array}{l}13,2 \\
53,4\end{array}$ & $\begin{array}{r}15,5 \\
8,9\end{array}$ \\
\hline $\begin{array}{l}\text { Nephrotisches } \\
\text { Syndrom }\end{array}$ & $\begin{array}{l}\text { a) } \\
\text { b) }\end{array}$ & $\begin{array}{r}14 \\
5\end{array}$ & $\begin{array}{l}39,1 \\
19,3\end{array}$ & $\begin{array}{l}8,0 \\
4,2\end{array}$ & $\begin{array}{l}20,0 \\
23,9\end{array}$ & $\begin{array}{l}15 ; 6 \\
50,7\end{array}$ & $\begin{array}{r}17,2 \\
4,6\end{array}$ \\
\hline
\end{tabular}

Bei entzündlicben Prozessen und malignen Erkrankungen sind auch mit dieser Methode keine wesentlichen Veränderungen der Lipoproteide nachzuweisen. Bei Lebererkrankungen findet sich nun nicht nur eine Abnahme der $\alpha$-Lipide, sondern auch der Eiweißanteil dieser Fraktion ist vermindert (11), so da $\beta$ die Abnahme der „Albumin-Fraktion" in der diese $\alpha$-Lipidfraktion mitwandert auch durch einen Verlust an Lipoproteiden verursacht ist. Das gleiche gilt für die $\alpha_{2}$-Fraktion. Sowohl bei der akuten Hepatitis, wie auch bei der Lebercirrhose sind die Lipoproteide dieser Fraktion erniedrigt. Dagegen sind - bei den hohen Werten für Protein- und Lipidanteile und der engen Korrelation $z$ wischen beiden - die Lipoproteide $\operatorname{der} \beta$-Fraktion erheblich vermehrt.

Ganz anders sind die Verhältnisse bei der.chronischen Hepatitis: hier sind die $\ddot{\alpha}_{1}$ - und $\alpha_{2}$-Lipoproteid-Fraktionen erhöht. Auffallend sind auch die hohen $\alpha_{2}$-Lipoproteidwerte bei Fällen von Leberepithelverfettung, die 
somit gewisse Parallelen zu den Befunden bei chronischer Hepatitis zeigen. Bemerkenswerterweise sind dabei auch die Protein- und die Kohlenhydratanteile erhöht. Prinzipiell die gleichen Befunde finden sich bei der Arteriosklerose. Hier ist der Gehalt an $\alpha_{2}$-Lipoproteiden hoch, die Fettklärung verzögert. Die hohe $\alpha_{2}$-LipoproteidFraktion ist dabei nicht nur durch Neutralfette, sondern auch durch Lipoproteide bedingt. Die Gruppe des nepbrotisclen Syndroms zeigt oft erniedrigte $\alpha_{1}$-Lipoproteidwerte und exzessiv hohe $\alpha_{2}$-Lipoproteide. Diese Zunahme ist sowohl durch Lipoproteide als auch durch Neutralfette verursacht, wie vergleichende Untersuchungen vor und nach Ultrazentrifugierung der Seren ergeben. Alle diese Befunde entsprechen somit denen, wie sie bei Fetttransportstörungen zu erheben sind.
Über den diagnostischen Wert hinaus haben diese Befunde auch eine aktuelle praktische Bedeutung, die in das Gebiet der parenteralen Ernährung mit Fettemulsionen hineinspielt. Nachdem brauchbare Präparate zur Verfügung stehen und die Indikationen für die parenterale Fetternährung abgegrenzt sind, ist es für den Kliniker von besonderem Interesse jene Fälle zu erfassen, bei denen eine parenterale Fetternährung kontraindiziert ist. Wie die Untersuchungen mit der Dünnschichtelektrophorese gezeigt haben, ist in allen Fällen in denen ein hoher $\alpha_{2}$-Lipoproteidgehalt des Nüchternserums nachweisbar ist, der Abtransport parenteral zugeführter Fette verzögert. Bisher vorliegende Untersuchungsergebnisse sprechen dafür, daß besonders Kranke mit Arteriosklerose, Leberepithelverfettung und mit nephrotischem Syndrom hiervon betroffen sind.

\section{Literatur}

1. Dittmar, A., Papierelektrophorese, Gustav Fischer, Jena (1962). - 2. Scheiffarth, F., G. Berg und H. Götz, Papierelektrophorese in der Klinik und Praxis, Urban \& Schwarzenberg, Berlin-München (1962). - 3. Swarn, B., Scand. J. Clin. Laborat. Invest. 4, 98 (1952). - 4. WunderLY, Ch., Die Papierclektrophorese, H. R. Sauerländer \& Co. Aarau und Frankfurt! Main (1954). - 5. Pezold, F. A. Lipide und Lipoproteide im Blutplasma, Springer-Verlag, Berlin-Göttingen-Heidelberg, (1961). - 6. Berg, G., F. Schetpfarth, C. J. EstLer und H. SchöN, Klin. Wschr. 36, 203 (1958). - 7. Schetrler, G., M. Eggstein und F. Dietrich, Klin. Wschr. 34, 684 (1956). - 8. Wercker, B., und I. WeICKER, Klin. Wschr. 33, 1028 (1955). 9. BerG., G, Med. und Ernährung, Sonderheft: Nahrungsfett und Atherosklerose 34 (1961). - 10. Berg, G., F. Scheiffarth und
G. Marwan, Klin. Wschr. 35, 415 (1957). - 11. Berg, G., L. R. Trabulsi und F. Scheiffarth, Klin. Wschr. 36, 202 (1958). 12. Berg, G. und H. Schön, Verh. Dtsch. Ges. inn. Med. 65, 715 (1959). - 13. Dietrich, F., Hoppe-Seyler's Z. physiol. Chem. 302, 327 (1955). - 14. Kunkel, G. und R. J. Slater, Proc. Soc. exp. Biol. Med. (N. Y.) 80, 42 (1952). - 15. Paroneto, F., Chung-IWANG und D. AdLersberg, Science 124, 1148 (1956). - 16. Berg, G. und E. Roller, Blut VI, 32 (1960). - 17. KunKel, G. und R. TrautmanN, J. Clin. Invest. 33, 641 (1956). - 18. Schettler, G. undF. Dietrich, M. EgGstein, Verh. dtsch. Ges. Kreisl.-forsch. 21, 124 (1955). 19. Berg, G., Fette, Seifen, Anstrichmittel 63, 329 (1961). - 20. Berg, G., Melsunger Med.-pharmaz. Mitt. 2315 (1961). - 21. Berg, G. und G. Finger, Med. Welt 48, 2521 (1961).
Dr. med. G. Berg

Medizinische Klinik der Universität Erlangen-Nürnberg 852 Erlangen, Krankenhausstr. 12 